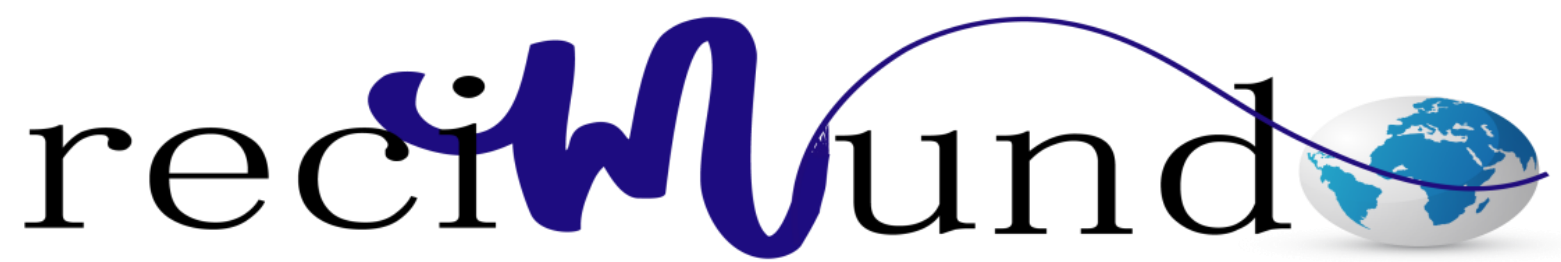

Revista Cientifica Mundo de la Investigación y el Conocimiento

Ricardo Antonio Mejía Aray a; Jonathan Paúl Durán González ${ }^{\text {b}}$; María Lucia Ortega Solórzano ${ }^{\text {c; }}$ Carlos Fernando Joza Aguayo ${ }^{\text {d; }}$ María Julia Larrea Villacis ${ }^{\text {e; }}$ Victor Enrique Vallejo Romero ${ }^{\mathrm{f}}$

Complicaciones de los pacientes sometidos a tratamiento de hemodiálisis Complications of patients undergoing hemodialysis treatment

Revista Científica Mundo de la Investigación y el Conocimiento. Vol. 3 núm.3. Esp., noviembre, ISSN: 2588-073X, 2019, pp. 1042-1056

DOI: $\underline{10.26820 / \text { recimundo/3.(3.Esp).noviembre.2019.1042-1056 }}$

URL: http://recimundo.com/index.php/es/article/view/648

Código UNESCO: 3205 Medicina Interna

Tipo de Investigación: Artículo de Revisión

(C) RECIMUNDO; Editorial Saberes del Conocimiento, 2019

Recibido: 15/09/2019

Aceptado: 23/10/2019

Publicado: 30/11/2019

Correspondencia: dr.ricardomejia@gmail.com

a. Médico Cirujano; Director Distrito de Salud 13D05; dr.ricardomejia@ gmail.com

b. Médico; Médico Residente de Emergencia - Centro Clínico Quirúrgico Hospital del Día IESS Jipijapa; jhonduran90@ hotmail.com

c. Médico Cirujano; Manadialisis S.A.; jorgitoylu@gmail.com

d. Médico Cirujano; Manadialisis S.A.; kafferjoza13@gmail.com

e. Médico; Médico Residente del IESS Portoviejo; julialarrea2345@ hotmail.com

f. Médico Cirujano; Médico Residente del IESS Portoviejo; victor-vallejo91@ @otmail.com 


\section{Complicaciones de los pacientes sometidos a tratamiento de hemodiálisis}

Vol. 3, núm. 3., (2019)

Ricardo Antonio Mejía Aray; Jonathan Paúl Durán González; María Lucia Ortega Solórzano; Carlos Fernando Joza Aguayo; María Julia Larrea Villacis; Victor Enrique Vallejo Romero

\section{RESUMEN}

La hemodiálisis es una de las terapias de reemplazo renal, además de la diálisis peritoneal y el trasplante renal. Aunque la hemodiálisis puede mejorar la morbilidad y la mortalidad en pacientes renales, tiene muchas complicaciones que ocurren durante las sesiones de diálisis y / o el uso a largo plazo de la hemodiálisis. De esta manera y pesar de los beneficios para los pacientes, la hemodiálisis implica varias complicaciones relacionadas con la eliminación de solutos urémicos, agua y electrolitos. Estas complicaciones pueden aumentar la morbilidad y mortalidad de pacientes renales en tratamiento de hemodiálisis. Entre las complicaciones comunes que se producen durante la diálisis se encuentran la hipotensión, la hipoglucemia, los trastornos electrolíticos, el síndrome de desequilibrio, la hipocalcemia, entre otros. En la diálisis a largo plazo, hay complicaciones como amiloidosis, enfermedad ósea, trastornos endocrinos, infección, complicaciones cardiovasculares, acceso vascular y complicaciones nutricionales. Durante el desarrollo del proceso investigativo se presentan las complicaciones más importantes en pacientes sometidos a tratamiento de hemodiálisis, a través de una revisión bibliográfica que brinda información de interés acerca del tema.

Palabras Claves: Hemodiálisis, hipotensión, calambres musculares, transmisión de infecciones, amiloidosis, complicaciones de la EH. 


\title{
Complicaciones de los pacientes sometidos a tratamiento de hemodiálisis
}

Vol. 3, núm. 3., (2019)

Ricardo Antonio Mejía Aray; Jonathan Paúl Durán González; María Lucia Ortega Solórzano; Carlos Fernando Joza Aguayo; María Julia Larrea Villacis; Victor Enrique Vallejo Romero

\begin{abstract}
Hemodialysis is one of the renal replacement therapies, in addition to peritoneal dialysis and renal transplantation. Although hemodialysis can improve morbidity and mortality in renal patients, it has many complications that occur during dialysis sessions and / or long-term use of hemodialysis. In this way and despite the benefits for patients, hemodialysis involves several complications related to the elimination of uremic solutes, water and electrolytes. These complications may increase the morbidity and mortality of renal patients on hemodialysis treatment. Among the common complications that occur during dialysis are hypotension, hypoglycemia, electrolyte disorders, imbalance syndrome, hypocalcemia, among others. In long-term dialysis, there are complications such as amyloidosis, bone disease, endocrine disorders, infection, cardiovascular complications, vascular access and nutritional complications. During the development of the research process, the most important complications are presented in patients undergoing hemodialysis treatment, through a literature review that provides information of interest on the subject.
\end{abstract}

Key Words: Hemodialysis, Hypotension, Muscle Cramps, Infection Transmission, Amyloidosis, Complications of HD. 


\section{Complicaciones de los pacientes sometidos a tratamiento de hemodiálisis}

Vol. 3, núm. 3., (2019)

Ricardo Antonio Mejía Aray; Jonathan Paúl Durán González; María Lucia Ortega Solórzano; Carlos Fernando Joza Aguayo; María Julia Larrea Villacis; Victor Enrique Vallejo Romero

\section{Introducción.}

La terapia de reemplazo renal es una opción para tratar pacientes con insuficiencia renal aguda y / o crónica. Hay dos opciones de tratamiento para un paciente que desarrolla insuficiencia renal en etapa terminal con evidencia de síntomas de uremia. El trasplante de riñón es la mejor opción en el tratamiento de pacientes con insuficiencia renal en etapa terminal (ESRF), sin embargo, la escasez de órganos donados, el seguimiento previo y posterior al trasplante y la disponibilidad del equipo mundial de trasplante son obstáculos y dificultades en muchos países. Estas razones han llevado a otras alternativas de la modalidad de terapia de reemplazo renal, que es la diálisis, particularmente la hemodiálisis.

La causa subyacente de la enfermedad renal en etapa terminal (ESRD) está cambiando cada vez más. En los Estados Unidos, alrededor del 40\% de los pacientes tienen ERT debido a diabetes, hipertensión, infección recurrente del tracto urinario, uso de drogas, glomerulopatías, enfermedades vasculíticas, etc. La edad promedio de los pacientes que comienzan la diálisis ha aumentado a 64 años. Este aumento en la edad de inicio de la diálisis ha llevado a enfermedades más coexistentes que se asocian con el envejecimiento y también con las complicaciones de la diálisis. (Locatelli, Manzoni, Del Vecchio, \& Di Filippo, 2015)

El autor (Blagg, 2014) expresa que la modalidad de reemplazo renal comúnmente utilizada es la hemodiálisis (HD). La HD para ESRD comenzó en humanos en 1960 con el desarrollo de la derivación arteriovenosa de teflón por Scribner y compañeros de trabajo en la Universidad de Washington. El número de pacientes aumentó constantemente, hasta el año 2002, alrededor de 250,000 en los Estados Unidos y más de 500,000 pacientes en todo el mundo estaban en diálisis.

Las complicaciones a largo plazo más frecuentes en pacientes con EH están relacionadas con enfermedades cardiovasculares, amiloidosis por $\beta$-microglobulina ( $(\mathrm{M})$, osteodistrofia renal, acceso vascular, desnutrición y transmisión de infecciones en pacientes dependientes de EH. 


\section{Complicaciones de los pacientes sometidos a tratamiento de hemodiálisis}

Vol. 3, núm. 3., (2019)

Ricardo Antonio Mejía Aray; Jonathan Paúl Durán González; María Lucia Ortega Solórzano; Carlos Fernando Joza Aguayo; María Julia Larrea Villacis; Victor Enrique Vallejo Romero

\section{Métodos y Materiales.}

Para el desarrollo de este proceso investigativo, se plantea como metodología la encaminada hacia una orientación científica particular que se encuentra determinada por la necesidad de indagar en forma precisa y coherente una situación, en tal sentido (Davila, 2015) define la metodología "como aquellos pasos previos que son seleccionados por el investigador para lograr resultados favorables que le ayuden a plantear nuevas ideas”. (p.66)

Lo citado por el autor, lleva a entender que el desarrollo de la acción investigativa busca simplemente coordinar acciones enmarcadas en una revisión bibliográfica con el fin de complementar ideas previas relacionadas Complicaciones de los pacientes sometidos a tratamiento de Hemodiálisis, a través de una revisión de literatura, para así finalmente elaborar un cuerpo de consideraciones generales que ayuden a ampliar el interés propuesto.

\section{Tipo de Investigación.}

Dentro de toda práctica investigativa, se precisan acciones de carácter metodológico mediante las cuales, se logra conocer y proyectar los eventos posibles que la determinan, así como las características que hacen del acto científico un proceso interactivo ajustado a una realidad posible de ser interpretada. En este sentido, se puede decir, que la presente investigación corresponde al tipo documental, definido por Castro (2016), "se ocupa del estudio de problemas planteados a nivel teórico, la información requerida para abordarlos se encuentra básicamente en materiales impresos, audiovisuales y /o electrónicos”. (p.41).

En consideración a esta definición, la orientación metodológica permitió la oportunidad de cumplir con una serie de actividades inherentes a la revisión y lectura de diversos documentos donde se encontraron ideas explicitas relacionadas con los tópicos encargados de identificar a cada característica insertada en el estudio. Por lo tanto, se realizaron continuas interpretaciones con el claro propósito de revisar aquellas apreciaciones o investigaciones propuestas por diferentes investigadores relacionadas con el tema de interés, para luego dar la respectiva argumentación a los planteamientos, en función a las necesidades encontradas en la indagación. 


\section{Complicaciones de los pacientes sometidos a tratamiento de hemodiálisis}

Vol. 3, núm. 3., (2019)

Ricardo Antonio Mejía Aray; Jonathan Paúl Durán González; María Lucia Ortega Solórzano; Carlos Fernando Joza Aguayo; María Julia Larrea Villacis; Victor Enrique Vallejo Romero

\section{Fuentes Documentales.}

El análisis correspondiente a las características que predomina en el tema seleccionado, llevan a incluir diferentes fuentes documentales encargadas de darle el respectivo apoyo y en ese sentido cumplir con la valoración de los hechos a fin de generar nuevos criterios que sirven de referencia a otros procesos investigativos. Para (CASTRO, 2016) las fuentes documentales incorporadas en la investigación documental o bibliográfica, "representa la suma de materiales sistemáticos que son revisados en forma rigurosa y profunda para llegar a un análisis del fenómeno". (p.41). Por lo tanto, se procedió a cumplir con la realización de una lectura previa determinada para encontrar aquellos aspectos estrechamente vinculados con el tema, con el fin de explicar mediante un desarrollo las respectivas apreciaciones generales de importancia.

\section{Técnicas para la Recolección de la Información.}

La conducción de la investigación para ser realizada en función a las particularidades que determinan a los estudios documentales, tiene como fin el desarrollo de un conjunto de acciones encargadas de llevar a la selección de técnicas estrechamente vinculadas con las características del estudio. En tal sentido, (Bolívar, 2015), refiere, que es "una técnica particular para aportar ayuda a los procedimientos de selección de las ideas primarias y secundarias”. (p. 71).

Por ello, se procedió a la utilización del subrayado, resúmenes, fichaje, como parte básica para la revisión y selección de los documentos que presentan el contenido teórico. Es decir, que mediante la aplicación de estas técnicas se pudo llegar a recoger informaciones en cuanto a la revisión bibliográfica de los diversos elementos encargados de orientar el proceso de investigación. Tal como lo expresa, (Bolívar, 2015) "las técnicas documentales proporcionan las herramientas esenciales y determinantes para responder a los objetivos formulados y llegar a resultados efectivos" (p. 58). Es decir, para responder con eficiencia a las necesidades investigativas, se introdujeron como técnica de recolección el método inductivo, que hizo posible llevar a cabo una valoración de los hechos de forma particular para llegar a la explicación desde una visión general.

Asimismo, se emplearon las técnicas de análisis de información para la realización de la investigación que fue ejecutada bajo la dinámica de aplicar diversos elementos encargados de 


\section{Complicaciones de los pacientes sometidos a tratamiento de hemodiálisis}

Vol. 3, núm. 3., (2019)

Ricardo Antonio Mejía Aray; Jonathan Paúl Durán González; María Lucia Ortega Solórzano; Carlos Fernando Joza Aguayo; María Julia Larrea Villacis; Victor Enrique Vallejo Romero

determinar el camino a recorrer por el estudio, según, (Bolívar, 2015) las técnicas de procesamiento de datos en los estudios documentales "son las encargadas de ofrecer al investigador la visión o pasos que debe cumplir durante su ejercicio, cada una de ellas debe estar en correspondencia con el nivel a emplear" (p. 123). Esto indica, que para llevar a cabo el procesamiento de los datos obtenidos una vez aplicado las técnicas seleccionadas, tales como: fichas de resumen, textual, registros descriptivos entre otros, los mismos se deben ajustar al nivel que ha sido seleccionado.

\section{Resultados}

\section{Enfermedad cardiovascular (ECV).}

Este tipo de patología es la principal causa de muerte en pacientes con ESRD en HD regular. La aterosclerosis está presente en la mayoría de los pacientes de diálisis a largo plazo, si no en todos. La ECV en pacientes con EH es de 5 a 10 veces mayor que en la población general, y representa al menos la mitad de las muertes de todos los pacientes. La muerte en estos pacientes se debe principalmente a la enfermedad coronaria (CHD). El diagnóstico de CHD en pacientes dializados crónicos requiere cirugía de derivación de la arteria coronaria que tiene un aumento de más de tres veces en la tasa de mortalidad en pacientes con ERC que en la población general. (Liu \& Sanders, 2013)

La hipertensión no es común en pacientes con EH y, por lo general, es difícil de controlar debido a muchos factores. La hipertensión mal controlada es un factor de riesgo importante para CVD.

El autor (Raggi, 2011) afirma que otros factores de riesgo que pueden aumentar la posibilidad de cardiopatía coronaria son la hiperfosfatemia y el producto elevado de calcio y fósforo con depósito de calcio en las arterias coronarias, el sistema de conducción cardíaca, las válvulas cardíacas y los vasos sanguíneos en otros lugares. La anemia, la hipertrigliceridemia baja el colesterol HDL aumenta la lipoproteína, la deficiencia o resistencia a la insulina, la alta homocisteína en la sangre, la disfunción endotelial, la inflamación y la proteína C reactiva elevada, y fumar son comunes, y aumentan significativamente el riesgo de CHD. Muchos, si no todos, estos 


\section{Complicaciones de los pacientes sometidos a tratamiento de hemodiálisis}

Vol. 3, núm. 3., (2019)

Ricardo Antonio Mejía Aray; Jonathan Paúl Durán González; María Lucia Ortega Solórzano;

Carlos Fernando Joza Aguayo; María Julia Larrea Villacis; Victor Enrique Vallejo Romero

factores de riesgo pueden haber ocurrido durante los años de insuficiencia renal crónica antes incluso de comenzar la HD. La reducción de las complicaciones CVD en ESRD se puede lograr modificando estos Factores de Riesgo.

El control de la hipertensión por drogas es muy necesario (Scribner, 2009). Se recomienda una HD adecuada y prolongada, y debe llevarse a cabo para controlar la hipertensión en pacientes con HD. La reducción de la concentración de colesterol LDL es de gran importancia para minimizar el riesgo de infarto de miocardio en pacientes no renales y con enfermedad coronaria. La vitamina $\mathrm{E}$ ha sido sugerida como un medio para reducir el estrés oxidativo y la mortalidad cardiovascular. Sin embargo, los autores (Isotalo, Halil, Green, Tang, Lach, \& Veinot, 2012) afirman que lograr un nivel más bajo de colesterol LDL en plasma con Cerivastain que también tiene un efecto antiinflamatorio puede reducir las complicaciones a largo plazo de la EH en pacientes con $\mathrm{EH}$.

La hiperfosfatemia y el producto de fosfato de calcio elevado, y el uso de ligantes de fosfato a base de calcio son factores de alto riesgo para la calcificación vascular. El bajo contenido de dializado de calcio y el uso selectivo de análogos de vitamina D ayuda a minimizar y mejorar las complicaciones de calcificación ósea y vascular. (Raggi, 2011)

La anemia es una complicación más común en pacientes con EH. Es un factor de riesgo importante para la ECV y el aumento de la mortalidad en pacientes con EH. La corrección de la anemia mediante suplementos tónicos adecuados, eritropoyetina y pacientes con EH adecuados mejora la dinámica cardiovascular, la tolerancia al ejercicio y la morbilidad, así como la mortalidad.

La enfermedad cardíaca en la ESRD dependiente de HD generalmente se maneja con medidas de tratamiento ordinario de problemas cardíacos, sin embargo, la angioplastia coronaria transluminal percutánea, la colocación de stent y la cirugía de derivación están indicadas en pacientes con HD. Estas medidas de intervención tienen mayor riesgo de mortalidad, recurrencia y reestenosis que la población general.

\section{Amilosis.}




\section{Complicaciones de los pacientes sometidos a tratamiento de hemodiálisis}

Vol. 3, núm. 3., (2019)

Ricardo Antonio Mejía Aray; Jonathan Paúl Durán González; María Lucia Ortega Solórzano; Carlos Fernando Joza Aguayo; María Julia Larrea Villacis; Victor Enrique Vallejo Romero

La deposición de amiloide $\beta \mathrm{M}$ generalmente comienza durante algunos meses después de comenzar la HD. Los hallazgos clínicos y radiológicos de amiloide aparecen generalmente después de cinco años o más de HD. La articulación esternoclavicular y de cadera son comúnmente las primeras articulaciones involucradas, sin embargo, cualquier articulación puede verse afectada. La compresión severa de la médula espinal debido al depósito de amiloidosis $\beta \mathrm{M}$ ocurre a menudo después de diez años de HD (Floege \& Kettele, 2011). El depósito de amiloidosis en las articulaciones puede presentarse clínicamente por características de periartritis de los hombros, síndrome del túnel carpiano, tenosinovitis flexora de las manos, rigidez, dolor e hinchazón de otras articulaciones, depósitos debajo de la piel y espondiloartropatía. Después de quince años de HD regular, Se produce una infiltración sistémica de amiloide $\beta \mathrm{M}$ en los órganos viscerales, pero generalmente es asintomática.

La patología de la amiloidosis $\beta$ M no está bien establecida y se conoce poco. Se informa que, después de un trasplante renal exitoso, los síntomas de amiloide mejoran y su progresión se detiene con la reducción del plasma de amiloide $\beta$. Otros han informado que las modalidades de HD disponibles pueden eliminar parte de la $\beta \mathrm{M}$ producida, pero no reducirán la cantidad de amiloide $\beta \mathrm{M}$ que puede prevenir las complicaciones conocidas de la amiloidosis, incluso con diálisis de alto flujo utilizando agua ultrapura para producir dializado. (Cannata, 2014)

\section{Osteodistrofia.}

La HD a largo plazo aumenta el riesgo de osteodistrofia. La osteodistrofia se asocia comúnmente con hiperparatiroidismo secundario que se caracteriza por una hormona paratiroidea plasmática alta a pesar del calcio y fosfato séricos normales. (Coco \& Rush)

La prevalencia de osteodistrofia en pacientes con $\mathrm{EH}$ a largo plazo, junto con un manejo y amortiguamiento de las cargas de calcio que acompañan al hiperparatiroidismo secundario está aumentando. La frecuencia de fracturas de cadera debido a osteodistrofia es más frecuente y grave en pacientes con EH a largo plazo que en la población general compatible. Las enfermedades óseas dinámicas ocurren en pacientes con ERC, pero su prevalencia es mayor después de comenzar la $\mathrm{EH}$. 


\section{Complicaciones de los pacientes sometidos a tratamiento de hemodiálisis}

Vol. 3, núm. 3., (2019)

Ricardo Antonio Mejía Aray; Jonathan Paúl Durán González; María Lucia Ortega Solórzano; Carlos Fernando Joza Aguayo; María Julia Larrea Villacis; Victor Enrique Vallejo Romero

\section{Desnutrición.}

La desnutrición es a menudo común en pacientes dependientes de HD, y la duración del tiempo en diálisis es un fuerte predictor de desnutrición. Los pacientes con HD por más de diez años tienden a perder su peso corporal a pesar de la ingesta adecuada de proteínas (Fukagawa, 2013). Aunque la causa subyacente de la desnutrición no está clara, puede deberse a una acidosis metabólica crónica o a una disminución de la actividad física como resultado de $\beta \mathrm{M}$-amiloidosis en lugar de la reducción del gasto energético. Se informó que la tasa de supervivencia se correlaciona con los niveles de albúmina sérica y prealbúmina. La aparición de enfermedad ósea adinámica también parece relacionarse con la desnutrición, aunque no está claro si se trata de una relación causal o si ambas condiciones son características de los sobrevivientes de HD a largo plazo. Se ha informado de deficiencia de zinc y selenio en pacientes con diálisis crónica, que es principalmente debido a la desnutrición y la ósmosis inversa, el tratamiento del agua puede tener un papel.

Acceso vascular.

El acceso vascular de la EH debe planificarse lo antes posible, especialmente en pacientes de edad avanzada y diabéticos. La fístula arteriovenosa (AV) nativa siempre se recomienda siempre que sea posible (Ellis, 2018). Debe iniciarse la diálisis temprana, mientras reduce la frecuencia de complicaciones, pero puede o no mejorar la supervivencia del paciente durante los primeros meses de tratamiento. Las complicaciones del acceso vascular como infección, mal funcionamiento, estenosis, desfiguración, etc. son una preocupación importante y pueden conducir a una diálisis inadecuada.

La infección de acceso vascular por hemodiálisis es una causa común de hospitalización en pacientes con EH y puede causar complicaciones graves. En infecciones de injerto y catéter menos graves, a veces es posible erradicar la infección con antibióticos durante varias semanas. La persistencia de fiebres, un número elevado de glóbulos blancos en la sangre o síntomas constitucionales pueden indicar un fracaso del tratamiento con antibióticos, y eso indica que se debe eliminar el acceso vascular. (Woods \& Port, 2015) 


\section{Complicaciones de los pacientes sometidos a tratamiento de hemodiálisis}

Vol. 3, núm. 3., (2019)

Ricardo Antonio Mejía Aray; Jonathan Paúl Durán González; María Lucia Ortega Solórzano; Carlos Fernando Joza Aguayo; María Julia Larrea Villacis; Victor Enrique Vallejo Romero

\section{Transmisión de la infección.}

La diálisis ha llevado a una disminución de la mortalidad y la morbilidad en pacientes con ESRD, pero predispone a estos pacientes a infecciones nacidas en la sangre, en parte debido a una función anormal del sistema inmune en pacientes con ERC y en pacientes con ESRD dependientes de diálisis. En pacientes en diálisis, el recuento de glóbulos blancos puede ser normal, pero los glóbulos blancos generalmente no funcionan normalmente. Esta anormalidad ha llevado a un mayor riesgo de infección por estafilococos.

Los linfocitos participan principalmente en la protección contra infecciones causadas por virus y hongos. Los pacientes dependientes de HD tienen una mayor incidencia de infecciones virales y fúngicas debido al mal funcionamiento de los linfocitos. Infecciones virales simples como la gripe ocurre, pero también las condiciones graves como el herpes zóster y la hepatitis no son comunes. Por lo tanto, las vacunas contra estas infecciones disminuyen la gravedad de estas infecciones virales.

La propagación de agentes infecciosos desde la máquina de diálisis u otros procedimientos relacionados con la diálisis al paciente es inusual en el contexto de las políticas actuales de control de infecciones, pero rara vez ocurre. El líquido de dializado puede estar contaminado con un agente infeccioso y, en teoría, puede transmitir infecciones, aunque el material de membrana del dializador es una barrera eficaz contra la propagación de estos agentes desde la máquina a la sangre del paciente. La infección relacionada con el tratamiento de diálisis es inusual, pero puede ocurrir durante el proceso de colocación o extracción donde se pueden introducir agentes infecciosos en el acceso vascular del paciente debido a una técnica estéril inadecuada. Por lo tanto, es importante que los pacientes se familiaricen y él / ella debe insistir en que se debe utilizar una técnica estéril durante el inicio y el final del tratamiento de diálisis.

Varios estudios han demostrado una mayor prevalencia de VHB, VHC y VIH en pacientes con EH que en la población general. El aumento de la infección por estos virus puede deberse al uso compartido de máquinas de diálisis, la preparación inadecuada de fármacos parenterales y los métodos de control de infección inadecuados en las unidades de diálisis. Las necesidades de 


\section{Complicaciones de los pacientes sometidos a tratamiento de hemodiálisis}

Vol. 3, núm. 3., (2019)

Ricardo Antonio Mejía Aray; Jonathan Paúl Durán González; María Lucia Ortega Solórzano; Carlos Fernando Joza Aguayo; María Julia Larrea Villacis; Victor Enrique Vallejo Romero

transfusión de sangre y la inmunidad suprimida de los pacientes también pueden tener un papel en la transmisión de estos virus. (Alavian, 2011)

Los pacientes infectados por el VHC generalmente son asintomáticos. Esto está llevando a un mayor riesgo de transmisión del VHC porque estos pacientes y el personal de HD no estaban al tanto del riesgo de transmisión de la infección, y esto puede no hacer que cumplan estrictamente con las medidas estándar de infección. Se ha sugerido que la transmisión del VHC en las unidades de diálisis es principalmente el resultado de la contaminación ambiental, por lo tanto, actualmente no se recomiendan máquinas dedicadas para pacientes con VHC positivo. Además, debido a la falta de una vacuna eficaz contra el VHC, ha aumentado el riesgo de hepatitis crónica por VHC en pacientes con EH.

En un estudio realizado por (Raiza \& Alfredo, 2009) se determinó una prevalencia promedio determinada de VHC en pacientes de hemodiálisis en la ciudad de México, la prevalencia fue del 13.2\%, mientras que otros informaron una menor prevalencia. Adicional a eso (Raiza \& Alfredo, 2009) también informaron que la tasa promedio de infección por VHC en pacientes con EH en 12 áreas en México se ha estimado en aproximadamente un 7,6\%.

La prevalencia de las infecciones por el virus de la hepatitis parece ser mayor en los países subdesarrollados y en desarrollo que en los países desarrollados. Se ha informado que la prevalencia de VHB y VHC en Palestina es de $8.1 \%$ y $22 \%$ respectivamente. En Yemen, la tasa de prevalencia del VHC fue del 62,7\%. En el Medio Oriente, la tasa de infección por VHC en pacientes con EH es más alta; en Jordania, Siria (28\%, 48\% respectivamente). Se ha observado una alta incidencia de VHC en algunos países como India, donde se informa una prevalencia del $83 \%$ en pacientes en diálisis, una prevalencia del $71 \%$ en Venezuela y un $46 \%$ en Arabia Saudita. Se observó una baja prevalencia del 5,72\% en Suiza, mientras que en Francia fue del 7,7\%. Estas diferencias pueden deberse al período de tiempo transcurrido en la hemodiálisis, a la disponibilidad de la máquina de diálisis y al cumplimiento estricto por parte del personal y los pacientes de las normas de control de infecciones. 


\section{Complicaciones de los pacientes sometidos a tratamiento de hemodiálisis}

Vol. 3, núm. 3., (2019)

Ricardo Antonio Mejía Aray; Jonathan Paúl Durán González; María Lucia Ortega Solórzano; Carlos Fernando Joza Aguayo; María Julia Larrea Villacis; Victor Enrique Vallejo Romero

\section{Conclusiones}

Durante el desarrollo del proceso investigativo, se observó que debido a las complicaciones que se presentan en los pacientes sometidos a hemodiálisis, la prevención de la ERC y sus consecuencias tardías debe ser el objetivo final de los proveedores de atención médica. Con la finalidad de mejorar la supervivencia y reducir la morbilidad en todos los pacientes con ERC, es esencial optimizar la atención pre-ESRD de pacientes con insuficiencia renal crónica.

Adicional a esto se debe tener en cuenta los diversos factores que pueden afectar la morbilidad y la mortalidad de los pacientes con EH y el aumento constante de pacientes con ERC en HD en todo el mundo, está claro que existe una gran necesidad de más investigación para asignar la mejor manera de diagnosticar y tratar enfermedades renales en etapas tempranas para prevenir complicaciones tardías de ERC y HD. Se necesitan más investigaciones colaborativas de resultados multicéntricos nacionales e internacionales para establecer las pautas preventivas y de tratamiento que pueden ser útiles y afectar positivamente la morbilidad y la mortalidad en las enfermedades renales y las complicaciones a largo plazo de la diálisis.

\section{Bibliografía.}

Alavian, S. (2011). Prevalencia de infección por el virus de la hepatitis $C$ y factores de riesgo relacionados. Iran: Nefrología (Carlton).

Blagg, R. (2014). Long-term Complications in Hemodialysis. .

Bolívar, J. (2015). Investigación Documental. México. Pax.

Cannata, J. (2014). Adynamic bone and chronic renal failure: An overview. . Am J Med Sci, 8190.

Castro, J. (2016). Técnicas Documentales. México. Limusa.

Coco, M., \& Rush, H. (s.f.). Aumento de la incidencia de fracturas de cadera en pacientes en diálisis con bajo nivel de hormona paratiroidea en suero. . Am J Kidney Dis 2000. 


\section{Complicaciones de los pacientes sometidos a tratamiento de hemodiálisis}

Vol. 3, núm. 3., (2019)

Ricardo Antonio Mejía Aray; Jonathan Paúl Durán González; María Lucia Ortega Solórzano; Carlos Fernando Joza Aguayo; María Julia Larrea Villacis; Victor Enrique Vallejo Romero

Davila, A. (2015). Diccionario de Términos Cientificos. . Caracas: Editorial Oasis.

Ellis, P. (2018). Remisión tardía de insuficiencia renal terminal. QJM -

Floege, J., \& Kettele, r. M. (2011). Beta2-microglobulinderived amyloidosis: an update. Kidney Int, 45-67.

Fukagawa, M. (2013). Is aplastic osteodystrophy a disease of malnutrition? .

Isotalo, P., Halil, A., Green, M., Tang, A., Lach, B., \& Veinot, J. (2012). Metastatic calcification of the cardiac conduction system with heart block: an under-reported entity in chronic renal failure patients. J Forensic Sci 2000.

Liu, J., \& Sanders, J. (2013). Riesgos de morbilidad y mortalidad en pacientes en diálisis sometidos a cirugía de revascularización coronaria. Grupo de estudio de enfermedades cardiovasculares. Nueva Inglaterra: Circulation 2000.

Locatelli, F., Manzoni, C., Del Vecchio, L., \& Di Filippo, S. (2015). Changes in the clinical condition of haemodialysis patients. . J Nephrol .

Raggi, P. (2011). Detección y cuantificación de calcificaciones cardiovasculares con tomografía de haz de electrones para estimar el riesgo en pacientes en hemodiálisis. Nephrol 2000.

Raiza, H., \& Alfredo, P. (2009). Prevalencia y tendencias del virus de la inmunodeficiencia humana, el virus de la hepatitis $B$ y el virus de la hepatitis $C$ entre los donantes de sangre . Mexico.

Scribner, B. (2009). Can antihypertensive medication control BP in hemodialysis patients: yes or no?

Woods, J., \& Port, F. (2015). Clinical and biochemical correlates of starting "daily" hemodialysis. Kidney . 
Complicaciones de los pacientes sometidos a tratamiento de hemodiálisis Vol. 3, núm. 3., (2019)

Ricardo Antonio Mejía Aray; Jonathan Paúl Durán González; María Lucia Ortega Solórzano; Carlos Fernando Joza Aguayo; María Julia Larrea Villacis; Victor Enrique Vallejo Romero

$$
\text { (c) (i) (9) }
$$

\section{RECONOCIMIENTO-NOCOMERCIAL-COMPARTIRIGUAL}

CC BY-NC-SA

ESTA LICENCIA PERMITE A OTROS ENTREMEZCLAR, AJUSTAR Y CONSTRUIR A PARTIR DE SU OBRA CON FINES NO COMERCIALES, SIEMPRE Y CUANDO LE RECONOZCAN LA AUTORÍA Y SUS NUEVAS CREACIONES ESTÉN BAJO UNA LICENCIA CON LOS MISMOS TÉRMINOS. 